Pacific Journal of Mathematics

ISOMETRIES OF TRIDIAGONAL ALGEBRAS 


\title{
ISOMETRIES OF TRIDIAGONAL ALGEBRAS
}

\author{
Young Soo Jo
}

\begin{abstract}
Let $\operatorname{Alg} \mathscr{L}$ be a tridiagonal algebra which was introduced by $\mathrm{F}$. Gilfeather and D. Larson. In this paper it is proved that if $\varphi: A \lg \mathscr{L} \rightarrow \operatorname{Alg} \mathscr{L}$ is a linear surjective isometry, then there exist unitary operators $W$ and $V$ such that $\varphi(A)=W A V$ for all $A \in$ $\operatorname{Alg} \mathscr{L}$.
\end{abstract}

Introduction. The study of reflexive, but not necessarily self-adjoint, algebras of Hilbert space operators has become one of the fastestgrowing specialties in operator theory. In this paper we study the linear surjective isometries of a certain class of reflexive algebras, which were introduced by F. Gilfeather, A. Hopenwasser and D. Larson [5]. These algebras have been found to be useful counterexamples to a number of plausible conjectures. In particular, these algebras have non-trivial cohomology [5], and they admit automorphisms which are not spatially implemented [2].

First we introduce the notation which is used in this paper. Let $\left\{e_{1}, e_{2}, \ldots, e_{2 n}\right\}$ and $\left\{e_{1}, e_{2}, \ldots\right\}$ be fixed bases of $2 n$-dimensional complex Hilbert space and separable infinite dimensional Hilbert space, respectively. If $x_{1}, x_{2}, \ldots, x_{k}$ are vectors in some Hilbert space, we denote by $\left[x_{1}, x_{2}, \ldots, x_{k}\right]$ the closed subspace spanned by the vectors $x_{1}, x_{2}, \ldots, x_{k}$.

Let $x$ and $y$ be two vectors in some Hilbert space. Then $(x, y)$ means the inner product of the vectors $x$ and $y$.

Let $H_{2 n}$ be $2 n$-dimensional Hilbert space. We denote by $\mathscr{L}_{2 n}$ the subspace lattice generated by the subspaces $\left[e_{1}\right],\left[e_{3}\right],\left[e_{5}\right], \ldots,\left[e_{2 n-1}\right]$, $\left[e_{1}, e_{2}, e_{3}\right],\left[e_{3}, e_{4}, e_{5}\right], \ldots,\left[e_{2 n-3}, e_{2 n-2}, e_{2 n-1}\right],\left[e_{1}, e_{2 n-1}, e_{2 n}\right]$.

By $\operatorname{Alg} \mathscr{L}_{2 n}=\Phi_{2 n}$ we mean the algebra of bounded operators which leave invariant all of the subspaces in $\mathscr{L}_{2 n}$. It is easy to see that all 
such operators have the matrix form

$$
\left[\begin{array}{cccccccc}
* & * & & & & & & * \\
& * & & & & & \\
& * & * & * & & & \\
& & * & & & \\
& & * & \cdot & & \\
& & & & . & & \\
& & & & & * \\
& & & & & *
\end{array}\right],
$$

where all non-starred entries are zero. Note that all diagonal operators and the identity operator $I$ lie in $\operatorname{Alg} \mathscr{L}_{2 n}$.

Let $H_{\infty}$ represent infinite-dimensional separable Hilbert space, and let $\mathscr{L}_{\infty}$ be the lattice of subspaces generated by $\left[e_{1}\right],\left[e_{3}\right],\left[e_{5}\right], \ldots$, $\left[e_{1}, e_{2}, e_{3}\right],\left[e_{3}, e_{4}, e_{5}\right], \ldots$

Let $\Phi_{\infty}=\operatorname{Alg} \mathscr{L}_{\infty}$ be the algebra of bounded operators leaving every subspace of $\mathscr{L}_{\infty}$ invariant. Matricially, such operators have the form

$$
\left[\begin{array}{ccccccc}
* & * & & & & & \\
& * & & & & & \\
& * & * & * & & \\
& & * & & \\
& & * & \cdot & & \\
& & & & \cdot & \\
& & & & & .
\end{array}\right],
$$

where all non-starred entries are zero.

By an isometry of an operator algebra $\Phi$ we mean a linear map $\varphi: \Phi \rightarrow \Phi$ such that $\|\varphi(A)\|=\|A\|$ for every $A$ in $\Phi$. We do not assume any algebraic properties for isometries, although the main theorem will imply that such properties may exist.

Let $i$ and $j$ be two non-zero natural numbers. Then $E_{i j}$ is the matrix whose $(i, j)$-component is 1 and all other entries are zero.

In this paper we will prove the following theorem.

THEOREM. Let $\varphi: \operatorname{Alg} \mathscr{L}_{2 n} \rightarrow \operatorname{Alg} \mathscr{L}_{2 n}$ be a surjective isometry and let $\varphi(I)=U$. Then $U$ and $U^{*}$ are in $\operatorname{Alg} \mathscr{L}_{2 n}$, and $U$ is unitary. Let $\varphi_{1}: \operatorname{Alg} \mathscr{L}_{2 n} \rightarrow \operatorname{Alg} \mathscr{L}_{2 n}$ be the surjective isometry defined by $\varphi_{1}(A)=$ $U^{*} \varphi(A)$ for all $A$ in $\operatorname{Alg} \mathscr{L}_{2 n}$. Then either $\varphi_{1}\left(\mathscr{L}_{2 n}\right)=\mathscr{L}_{2 n}$ or $\varphi_{1}\left(\mathscr{L}_{2 n}\right)=$ $\mathscr{L}_{2 n}^{\perp}$. If $\varphi_{1}\left(\mathscr{L}_{2 n}\right)=\mathscr{L}_{2 n}$, then there exists a unitary operator $W$ such that $\varphi_{1}(A)=W A W^{*}$ for all $A$ in $\operatorname{Alg} \mathscr{L}_{2 n}$. If $\varphi_{1}\left(\mathscr{L}_{2 n}\right)=\mathscr{L}_{2 n}$, then there exist a conjugation $J$ and a unitary operator $W$ such that $\varphi_{1}(A)=$ 
$J W A^{*} W^{*} J$ for all $A$ in $\operatorname{Alg} \mathscr{L}_{2 n}$. Let $\varphi: \operatorname{Alg} \mathscr{L}_{\infty} \rightarrow \operatorname{Alg} \mathscr{L}_{\infty}$ be a surjective isometry and let $\varphi(I)=U$; then $U$ and $U^{*}$ are in $\operatorname{Alg} \mathscr{L}_{\infty}$ and $U$ is unitary. Let $\varphi_{1}: \operatorname{Alg} \mathscr{L}_{\infty} \rightarrow \operatorname{Alg} \mathscr{L}_{\infty}$ be the surjective isometry defined by $\varphi_{1}(A)=U^{*} \varphi(A)$ for all $A$ in $\operatorname{Alg} \mathscr{L}_{\infty}$. Then $\varphi_{1}(I)=I, \varphi_{1}\left(E_{i i}\right)=E_{i i}$ for all $i(i=1,2, \ldots), \varphi_{1}\left(\mathscr{L}_{\infty}\right)=\mathscr{L}_{\infty}$, and there are diagonal unitary operators $W$ and $V$ such that $\varphi_{1}(A)=W A V$ for all $A$ in $\operatorname{Alg} \mathscr{L}_{\infty}$.

\section{Examples of isometries.}

EXAMPLE 1. Let the Hilbert space be separable with an orthonormal basis $\left\{e_{k}: k=1,2, \ldots\right\}$ and let $U$ be a diagonal unitary operator whose $(i, i)$-component is $u_{i i}$ such that $\left|u_{i i}\right|=1$ for all $i$. Define $\varphi: \operatorname{Alg} \mathscr{L}_{\infty} \rightarrow$ $\operatorname{Alg} \mathscr{L}_{\infty}$ by $\varphi(A)=U^{*} A U$ for all $A$ in $\operatorname{Alg} \mathscr{L}_{\infty}$. Then $\varphi$ is a surjective isometry such that $\varphi(I)=I$, the $(i, i)$-component of $\varphi(A)$ is the same as the $(i, i)$-component of $A$ and if $A=\left(a_{i j}\right)$ is in $\operatorname{Alg} \mathscr{L}_{\infty}$, then the $(2 i+1,2 i+1)$-component of $\varphi(A)$ is $u_{2 i+1,2 i+1} a_{2 i+1,2 i} a_{2 i, 2 i}$ and the $(2 i+1,2 i+2)$-component of $\varphi(A)$ is $u_{2 i+1,2 i+1} a_{2 i+1,2 i+2} a_{2 i+2,2 i+2}$.

In Examples 2 and 3, the Hilbert space is $2 n$-dimensional with an orthonormal basis $\left\{e_{1}, e_{2}, \ldots, e_{2 n}\right\}$.

EXAMPLe 2. Let $D_{n}$ be the $n \times n$ matrix with 1 the $(i, n-i+1)$ component $(i=1,2, \ldots, n)$ and 0 elsewhere. Let $U_{2 i+1}=D_{2 i+1} \oplus$ $D_{2 n-2 i-1}$. Define $\varphi: \operatorname{Alg} \mathscr{L}_{2 n} \rightarrow \operatorname{Alg} \mathscr{L}_{2 n}$ by $\varphi(A)=U_{2 i+1} A U_{2 i+1}^{*}$ for every $A$ in $\operatorname{Alg} \mathscr{L}_{2 n}$. It is straightforward to show that $U_{2 i+1} A U_{2 i+1}^{*}$ and $U_{2 i+1}^{*} A U_{2 i+1}$ are in $\operatorname{Alg} \mathscr{L}_{2 n}$ for every $A$ in $\operatorname{Alg} \mathscr{L}_{2 n}$. So $\varphi$ is a surjective isometry such that $\varphi(I)=I, \varphi\left(E_{11}\right)=E_{2 i+1,2 i+1}, \varphi\left(E_{22}\right)=$ $E_{2 i, 2 i}, \ldots, \varphi\left(E_{2 i-1,2 i-1}\right)=E_{33}, \varphi\left(E_{2 i, 2 i}\right)=E_{22}, \varphi\left(E_{2 i+1,2 i+1}\right)=E_{11}$, $\varphi\left(E_{2 i+2,2 i+2}\right)=E_{2 n, 2 n}, \varphi\left(E_{2 i+3,2 i+3}\right)=E_{2 n-1,2 n-1}, \ldots, \varphi\left(E_{2 n, 2 n}\right)=$ $E_{2 i+2,2 i+2}$. Moreover, it is easy to check that $\varphi\left(\mathscr{L}_{2 n}\right)=\mathscr{L}_{2 n}$.

EXAMPLE 3. We denote the identity on $n$-dimensional Hilbert space by $I_{n}$. Let

$$
V_{2 i+1}=\left[\begin{array}{cc}
0 & I_{2 i} \\
I_{2 n-2 i} & 0
\end{array}\right] .
$$

Then $V_{2 i+1}$ is a unitary operator. Define $\varphi: \operatorname{Alg} \mathscr{L}_{2 n} \rightarrow \operatorname{Alg} \mathscr{L}_{2 n}$ by $\varphi(A)=V_{2 i+1} A V_{2 i+1}^{*}$ for every $A$ in $\operatorname{Alg} \mathscr{L}_{2 n}$. It is straightforward to show that $V_{2 i+1} A V_{2 i+1}^{*}$ and $V_{2 i+1}^{*} A V_{2 i+1}$ are in $\operatorname{Alg} \mathscr{L}_{2 n}$ for every $A$ in $\operatorname{Alg} \mathscr{L}_{2 n}$. So $\varphi$ is a surjective isometry such that $\varphi(I)=I$, $\varphi\left(E_{11}\right)=E_{2 i+1,2 i+1}, \varphi\left(E_{22}\right)=E_{2 i+2,2 i+2}, \ldots, \varphi\left(E_{2 n-2 i, 2 n-2 i}\right)=E_{2 n, 2 n}$, $\varphi\left(E_{2 n-2 i+1,2 n-2 i+1}\right)=E_{11}, \varphi\left(E_{2 n-2 i+2,2 n-2 i+2}\right)=E_{22}, \ldots, \varphi\left(E_{2 n, 2 n}\right)=$ $E_{2 i, 2 i}$. Moreover, it is easy to check that $\varphi\left(\mathscr{L}_{2 n}\right)=\mathscr{L}_{2 n}$. 
Example 4. Let $\varphi: \operatorname{Alg} \mathscr{L}_{4} \rightarrow \operatorname{Alg} \mathscr{L}_{4}$ be defined by $\varphi(A)=A_{f}$ for every $A$ in $\operatorname{Alg} \mathscr{L}_{4}$, where if

$$
A=\left[\begin{array}{cccc}
a_{11} & a_{12} & 0 & a_{14} \\
0 & a_{22} & 0 & 0 \\
0 & a_{32} & a_{33} & a_{34} \\
0 & 0 & 0 & a_{44}
\end{array}\right] ; \quad \text { then } A_{f}=\left[\begin{array}{cccc}
a_{44} & a_{34} & 0 & a_{14} \\
0 & a_{33} & 0 & 0 \\
0 & a_{32} & a_{22} & a_{12} \\
0 & 0 & 0 & a_{11}
\end{array}\right] \text {. }
$$

Define $J: \mathbf{C}^{4} \rightarrow \mathbf{C}^{4}$ by $J\left(x_{1}, x_{2}, x_{3}, x_{4}\right)^{t}=\left(\overline{x_{4}}, \overline{x_{3}}, \overline{x_{2}}, \overline{x_{1}}\right)^{t}$ for every $\left(x_{1}, x_{2}, x_{3}, x_{4}\right)^{t}$ in $\mathbf{C}^{4}$.

Then $J$ is a conjugation; that is,

(1) $J$ is bijective.

(2) $J(x+y)=J x+J y$ for $x, y$ in $\mathbf{C}^{4}$.

(3) $J(\alpha x)=\bar{\alpha} J x$ for every $\alpha$ in $\mathbf{C}$ and every $x$ in $\mathbf{C}^{4}$.

(4) $J^{2}=I$.

(5) $(J x, y)=(J y, x)$ for $x, y$ in $\mathbf{C}^{4}$.

It is easy to check that $\varphi(A)=J A^{*} J ; \varphi$ is a surjective isometry by (5) and $\varphi(I)=I$. This isometry is not implemented by any unitary operator. The algebra $\operatorname{Alg} \mathscr{L}_{2 n}$ admits this kind of isometry for other values of $n$. Note that in this example, if $E$ is in $\mathscr{L}_{2 n}$, then $\varphi(E)^{\perp}$ is in $\mathscr{L}_{2 n}$, that is, $\varphi\left(\mathscr{L}_{2 n}\right)=\mathscr{L}_{2 n}^{\perp}$.

2. General theorems. We want to show that every surjective isometry on $\operatorname{Alg} \mathscr{L}_{2 n}$ or $\operatorname{Alg} \mathscr{L}_{\infty}$ is a composition of the types mentioned in the examples. Our first task is to show that the image of the identity under a surjective isometry of $\operatorname{Alg} \mathscr{L}_{2 n}$ (or $\operatorname{Alg} \mathscr{L}_{\infty}$ ) must be a unitary operator.

Let $x$ and $y$ be two non-zero vectors in a Hilbert space $H$. Then $x^{*} \otimes y$ is a rank one operator defined by $x^{*} \otimes y(h)=(h, x) y$ for every $h$ in $H$.

LEMMA 1 (Longstaff [9]). Let $\mathscr{L}$ be a commutative lattice and let $x$ and $y$ be two vectors. Then $x^{*} \otimes y$ is in $\mathrm{Alg} \mathscr{L}$ if and only if there exists $E$ in $\mathscr{L}$ such that $y$ is in $E$ and $x$ is in $E_{-}^{\perp}\left(E_{-}^{\perp}\right.$ means $\left.\left(E_{-}\right)^{\perp}\right)$, where $E_{-}=V\{F: F$ is in $\mathscr{L}$ and $F \geq E\}$.

The following lemma appears in an unpublished paper. We include the proof for the convenience of the reader.

LEMMA 2 (Moore and Trent [10]). Let $\varphi: \operatorname{Alg} \mathscr{L}_{2 n} \rightarrow \operatorname{Alg} \mathscr{L}_{2 n}$ be a linear surjective isometry. If $A=\varphi(I)$ and if $x^{*} \otimes x$ is in $\operatorname{Alg} \mathscr{L}_{2 n}$, then $\|A x\|=\|x\|$. 
Proof. Without loss of generality, we may assume that $\|x\|=1$. Since $x^{*} \otimes A x=A\left(x^{*} \otimes x\right)$, the operator $x^{*} \otimes A x$ lies in $\operatorname{Alg} \mathscr{L}_{2 n}$, and there is an operator $R$ in $\operatorname{Alg} \mathscr{L}_{2 n}$ for which $\varphi(R)=x^{*} \otimes A x$. For any complex $\alpha$,

$$
\begin{aligned}
\|I+\alpha R\|^{2} & =\left\|A+\alpha\left(x^{*} \otimes A x\right)\right\|^{2} \\
& =\left\|\left(A+\alpha\left(x^{*} \otimes A x\right)\right)\left(A^{*}+\bar{\alpha}\left((A x)^{*} \otimes x\right)\right)\right\| \\
& =\left\|A A^{*}+\left(2 \operatorname{Re} \alpha+|\alpha|^{2}\right)\left((A x)^{*} \otimes A x\right)\right\| \\
& \leq 1+\left.\|A x\|^{2}|2 \operatorname{Re} \alpha+| \alpha\right|^{2} \mid .
\end{aligned}
$$

By choosing $\alpha=-i$ purely imaginary, and by letting $R=H+i K$ and $\delta \in \sigma(K)$, we find that $|1+t \delta|^{2} \leq 1+t^{2}\|A x\|^{2}$, or $\left(\|A x\|^{2}-\delta^{2}\right) t^{2}-2 \delta t \geq$ 0 for all real $t$, and it is easy to see that this condition implies that $\delta=$ 0 . Thus, $\sigma(K)=\{0\}, K=0$, and $R$ is Hermitian. Now let $\tau \in \sigma(R)$ and let $\alpha=t$ be real and deduce that $|1+t \tau|^{2} \leq 1+\|A x\|^{2}\left|2 t+t^{2}\right|$, or $2 t \tau+t^{2} \tau^{2} \leq\|A x\|^{2}\left|2 t+t^{2}\right|$. Choose $t=-2$ to get $\tau^{2} \leq \tau$, which means that $\tau \geq 0$ (and hence $R$ is a positive operator). Finally, let $t \rightarrow 0^{+}$and conclude that $\tau \leq\|A x\|^{2}$, and, consequently, that $\|R\| \leq\|A x\|^{2}$. But $\|R\|=\|\varphi(R)\|=\left\|x^{*} \otimes A x\right\|=\|x\|\|A x\|=\|A x\|$. Thus, $\|A x\| \leq\|A x\|^{2}$ and it follows that $\|A x\| \geq 1$. On the other hand, $\|A\|=1$, so $\|A x\|=1$ and we are done.

In particular, since $e_{i}^{*} \otimes e_{i}$ is in $\operatorname{Alg} \mathscr{L}_{2 n},\left\|A e_{i}\right\|=\left\|e_{i}\right\|=1$ by Lemma 2 for every $1 \leq i \leq 2 n$.

THEOREM 3. If $\varphi: \operatorname{Alg} \mathscr{L}_{2 n} \rightarrow \operatorname{Alg} \mathscr{L}_{2 n}$ is a surjective isometry, then $\varphi(I)$ is a unitary operator in $\mathrm{Alg} \mathscr{L}_{2 n}$.

Proof. Let $\varphi(I)=A=\left(a_{i j}\right)$. Then $\left|a_{i i}\right|=1$ by the above statement for all odd numbers $i ; 1 \leq i \leq 2 n$. But $\|A\|=\|I\|=1$, so $a_{12}=a_{1,2 n}=$ $0, a_{32}=a_{34}=0, a_{54}=a_{56}=0, \ldots, a_{2 n-1,2 n-2}=a_{2 n-1,2 n}=0$. Thus, $\varphi(I)=A$ is a diagonal matrix whose components have absolute value 1 and hence $A=\varphi(I)$ is a unitary operator in $\operatorname{Alg} \mathscr{L}_{2 n}$.

Similarly, we can get the following theorem.

THEOREM 4. If $\varphi: \operatorname{Alg} \mathscr{L}_{\infty} \rightarrow \operatorname{Alg} \mathscr{L}_{\infty}$ is a surjective isometry, then $\varphi(I)$ is a unitary operator in $\mathrm{Alg} \mathscr{L}_{\infty}$.

Let $\varphi(I)=U$. Then $U A$ and $U^{*} A$ are in $\operatorname{Alg} \mathscr{L}_{2 n}$ (resp. $\left.\operatorname{Alg} \mathscr{L}_{\infty}\right)$ if $A$ is in $\operatorname{Alg} \mathscr{L}_{2 n}$ (resp. $\operatorname{Alg} \mathscr{L}_{\infty}$ ). Define $\hat{\varphi}: \operatorname{Alg} \mathscr{L}_{2 n} \rightarrow \operatorname{Alg} \mathscr{L}_{2 n}$ by $\hat{\varphi}(A)=U^{*} \varphi(A)$ for every $A$ in $\operatorname{Alg} \mathscr{L}_{2 n}$ or $\hat{\varphi}: \operatorname{Alg} \mathscr{L}_{\infty} \rightarrow \operatorname{Alg} \mathscr{L}_{\infty}$ by 
$\hat{\varphi}(A)=U^{*} \varphi(A)$ for every $A$ in $\operatorname{Alg} \mathscr{L}_{\infty}$. Then $\hat{\varphi}$ is a surjective isometry such that $\hat{\varphi}(I)=I$.

Let $\Omega=\left\{A: A\right.$ is a diagonal matrix in $\operatorname{Alg} \mathscr{L}_{2 n}$ (or $\left.\left.\operatorname{Alg} \mathscr{L}_{\infty}\right)\right\}$. Then it is easy to check that $\Omega$ is the smallest von Neumann algebra containing $\mathscr{L}_{2 n}\left(\right.$ or $\left.\mathscr{L}_{\infty}\right)$ and $\Omega=\operatorname{Alg} \mathscr{L}_{2 n} \cap\left(\operatorname{Alg} \mathscr{L}_{2 n}\right)^{*}\left(\right.$ or $\left.\Omega=\operatorname{Alg} \mathscr{L}_{\infty} \cap\left(\operatorname{Alg} \mathscr{L}_{\infty}\right)^{*}\right)$.

We will require the following facts, first proved by Kadison.

LEMMA 5 (Kadison [8]). A linear map $\varphi$ of one $C^{*}$-algebra into another which carries the identity into the identity and is isometric on normal elements preserves adjoints, i.e., $\varphi\left(A^{*}\right)=(\varphi(A))^{*}$.

Definition 6. Let $\Phi_{1}$ and $\Phi_{2}$ be $C^{*}$-algebras. A Jordan isomorphism or $C^{*}$-isomorphism $\varphi: \Phi_{1} \rightarrow \Phi_{2}$ is a bijective linear map such that if $A$ is self-adjoint in $\Phi_{1}$, then $\varphi(A)$ is also self-adjoint in $\Phi_{2}$ and $\varphi\left(A^{n}\right)=(\varphi(A))^{n}$.

LEMma 7 (Kadison [8]). (a) A linear bijection $\varphi$ of one $C^{*}$-algebra $\Phi_{1}$ onto another $\Phi_{2}$ which is isometric is a $C^{*}$-isomorphism followed by left multiplication by a fixed unitary operator, viz, $\varphi(I)$.

(b) $A C^{*}$-isomorphism $\varphi$ of a $C^{*}$-algebra $\Phi_{1}$ onto a $C^{*}$-algebra $\Phi_{2}$ is isometric and preserves commutativity.

LEMMA 8. $\hat{\varphi}(\Omega)=\Omega$, (where $\hat{\varphi}$ and $\Omega$ are defined above).

Proof. Since $\hat{\varphi} \mid \Omega$ preserves adjoints by Lemma $5, \hat{\varphi}(\Omega)$ is contained in $\Omega$. Similarly, $\hat{\varphi}^{-1}(\Omega)$ is contained in $\Omega$. Hence $\hat{\varphi}(\Omega)=\Omega$.

Since $\hat{\varphi}: \operatorname{Alg} \mathscr{L}_{2 n} \rightarrow \operatorname{Alg} \mathscr{L}_{2 n}$ (or $\operatorname{Alg} \mathscr{L}_{\infty} \rightarrow \operatorname{Alg} \mathscr{L}_{\infty}$ ) is a surjective isometry, just like $\varphi$, and since the main theorem would be true of $\varphi$ if it were true of $\hat{\varphi}$, we now work exclusively with $\hat{\varphi}$ and drop the "^" symbol. Equivalently we assume that $\varphi(I)=I$.

Then we can get the following corollary.

CoROllaRY 9. If $\varphi: \operatorname{Alg} \mathscr{L}_{2 n} \rightarrow \operatorname{Alg} \mathscr{L}_{2 n}\left(\right.$ or $\left.\operatorname{Alg} \mathscr{L}_{\infty} \rightarrow \operatorname{Alg} \mathscr{L}_{\infty}\right)$ is a surjective isometry such that $\varphi(I)=I$, then $\varphi(\Omega)=\Omega$.

LeMMA 10. Let $\varphi: \operatorname{Alg} \mathscr{L}_{2 n} \rightarrow \operatorname{Alg} \mathscr{L}_{2 n}\left(\right.$ or $\left.\operatorname{Alg} \mathscr{L}_{\infty} \rightarrow \operatorname{Alg} \mathscr{L}_{\infty}\right)$ be a surjective isometry such that $\varphi(I)=I$. Then $E$ is a projection in $\Omega$ if and only if $\varphi(E)$ is a projection in $\Omega$.

Proof. First, suppose that $E$ is a projection in $\Omega$. Since $\varphi \mid \Omega$ is a Jordan isomorphism, $\varphi(E)=\varphi\left(E^{*}\right)=\varphi(E)^{*}$ and $\varphi(E)=\varphi\left(E^{2}\right)=$ 
$\varphi(E)^{2}$. So $\varphi(E)$ is a projection in $\Omega$ because $\varphi(\Omega)=\Omega$. Suppose that $\varphi(E)$ is a projection in $\Omega$. Then since $\varphi^{-1} \mid \Omega$ is a Jordan isomorphism, by the above argument $\varphi^{-1} \varphi(E)=E$ is a projection in $\Omega$.

LEMMA 11 (Kadison [8]). If $\varphi$ is a Jordan isomorphism from a $C^{*}$ algebra $\Phi_{1}$ onto a $C^{*}$-algebra $\Phi_{2}$, then $\varphi(B A B)=\varphi(B) \varphi(A) \varphi(B)$ with $A$ and $B$ in $\Phi_{1}$.

THEOREM 12. Let $\varphi: \operatorname{Alg} \mathscr{L}_{\infty} \rightarrow \operatorname{Alg} \mathscr{L}_{\infty}$ be a surjective isometry such that $\varphi(I)=I$. Let $\left\{e_{i}: i=1,2, \ldots\right\}$ be the orthonormal basis for which the generators of the lattice are $\left[e_{1}\right],\left[e_{3}\right], \ldots,\left[e_{2 n-1}\right], \ldots,\left[e_{1}, e_{2}, e_{3}\right]$, $\left[e_{3}, e_{4}, e_{5}\right], \ldots,\left[e_{2 n-3}, e_{2 n-2}, e_{2 n-1}\right], \ldots$ Then $\varphi\left(\left[e_{i}\right]\right)$ is rank-one for each $i ; i=1,2, \ldots$.

Proof. Let $E_{k}=\varphi^{-1}\left(\left[e_{k}\right]\right)$ for each $k ; k=1,2, \ldots$, that is, $\varphi\left(E_{k}\right)=$ [ $\left.e_{k}\right]$. Then $E_{k}$ is a projection in $\Omega$ by Lemma 10 . If $E_{k}$ is not a rank 1 projection, then $E_{k}=E+F$ with $E, F$ on $\operatorname{Alg} \mathscr{L}_{\infty}$, both non-zero projections. But then $\left[e_{k}\right]=\varphi^{-1}(E)+\varphi^{-1}(F)$ expresses $\left[e_{k}\right]$ as a sum of 2 non-zero projections.

With the same proof as Theorem 12, we can get the following theorem.

THEOREM 13. Let $\varphi: \operatorname{Alg} \mathscr{L}_{2 n} \rightarrow \operatorname{Alg} \mathscr{L}_{2 n}$ be a surjective isometry such that $\varphi(I)=I$. Then $\varphi\left(\left[e_{i}\right]\right)$ is rank-one in $\Omega$ for each $i$; $i=$ $1,2, \ldots, 2 n$.

LEMMA 14. Let $R$ be an operator and suppose that there is a nonnegative number $M$ and a positive number $N$ such that, for all complex numbers $\alpha$ with $|\alpha| \geq N$, we have $\|R+\alpha I\|^{2} \leq M^{2}+|\alpha|^{2}$. Then $R=0$.

Proof. Choose $x$ in the Hilbert space $H$, with $\|x\|=1$. We have $\|R x+\alpha x\|^{2} \leq M^{2}+|\alpha|^{2}$, or $\|R x\|^{2}+|\alpha|^{2}+2 \operatorname{Re} \bar{\alpha}(R x, x) \leq M^{2}+|\alpha|^{2}$, or $2 \operatorname{Re} \bar{\alpha}(R x, x) \leq M^{2}-\|R x\|^{2}$. Choosing $\alpha=\overline{t(R x, x)}$ for positive $t$, we get $2 t|(R x, x)|^{2} \leq M^{2}-\|R x\|^{2}$ for all $t>N$. This is impossible unless $(R x, x)=0$. The fact that this equation holds for all $x$ means that $R=0$.

LEMMA 15 (Moore and Trent [10]). Let $\varphi: \operatorname{Alg} \mathscr{L}_{2 n} \rightarrow \operatorname{Alg} \mathscr{L}_{2 n}$ (or $\left.\operatorname{Alg} \mathscr{L}_{\infty} \rightarrow \operatorname{Alg} \mathscr{L}_{\infty}\right)$ be a surjective isometry such that $\varphi(I)=I$. Let $P$ be 
a projection in $\Omega$ and let $T$ be in $\mathrm{Alg} \mathscr{L}_{2 n}$ (or $\left.\mathrm{Alg} \mathscr{L}_{\infty}\right)$ with $T=P T P^{\perp}$. Then we have $\varphi(T)=\varphi(P) \varphi(T) \varphi(P)^{\perp}+\varphi(P)^{\perp} \varphi(T) \varphi(P)$.

Proof. We begin by writing $\varphi(T)$ as $2 \times 2$ matrix, using the decomposition $I=\hat{P}+\hat{P}^{\perp}$ :

$$
\varphi(T)=\left[\begin{array}{ll}
R_{1} & R_{2} \\
R_{3} & R_{4}
\end{array}\right], \quad \hat{P}=\left[\begin{array}{ll}
I & 0 \\
0 & 0
\end{array}\right] \quad \text { and } \quad \hat{P}^{\perp}=\left[\begin{array}{ll}
0 & 0 \\
0 & I
\end{array}\right],
$$

where $\hat{P}=\varphi(P)$. Then, for all complex $\alpha$,

$$
\|T+\alpha P\|=\|\varphi(T)+\alpha \hat{P}\|=\left\|\left[\begin{array}{cc}
R_{1}+\alpha & R_{2} \\
R_{3} & R_{4}
\end{array}\right]\right\| .
$$

On the other hand, $T$, written using " $I=P+P^{\perp}$ ", is the matrix $T=\left[\begin{array}{ll}0 & S \\ 0 & 0\end{array}\right]$. So

$$
\begin{aligned}
\|T+\alpha P\|^{2} & =\left\|\left[\begin{array}{cc}
\alpha & S \\
0 & 0
\end{array}\right]\right\|^{2}=\left\|\left[\begin{array}{cc}
\alpha & S \\
0 & 0
\end{array}\right]\left[\begin{array}{cc}
\alpha & S \\
0 & 0
\end{array}\right]^{*}\right\| \\
& =\left\|\left[\begin{array}{cc}
0 & 0 \\
0 & |\alpha|^{2}+S S^{*}
\end{array}\right]\right\|=|\alpha|^{2}+\|S\|^{2}
\end{aligned}
$$

since $S S^{*}$ is a positive operator. Thus, $\left\|R_{1}+\alpha\right\|^{2} \leq|\alpha|^{2}+\|S\|^{2}$, and Lemma 14 tells us that $R_{1}=0$. Similarly, by considering $\left\|t+\alpha P^{\perp}\right\|$, we can show that $R_{4}=0$. So $\varphi(T)=\hat{P} \varphi(T) \hat{P}^{\perp}+\hat{P}^{\perp} \varphi(T) \hat{P}$.

TheOREM 16. Let $\varphi: \operatorname{Alg} \mathscr{L}_{\infty} \rightarrow \operatorname{Alg} \mathscr{L}_{\infty}$ be a surjective isometry such that $\varphi(I)=I$. Let $\varphi\left(E_{2 i-1,2 i-1}\right)=E_{j j}$ and let $\varphi\left(E_{2 i, 2 i}\right)=E_{k k}$. Then $|k-j|=1$.

Proof. Since

$$
\begin{gathered}
E_{2 i, 2 i}^{\perp} E_{2 i-1,2 i} E_{2 i, 2 i}=E_{2 i-1,2 i} \text { and } \\
E_{2 i-1,2 i-1} E_{2 i-1,2 i} E_{2 i-1,2 i-1}^{\perp}=E_{2 i-1,2 i},
\end{gathered}
$$

Lemma 15 tells us that

$$
\begin{aligned}
\varphi\left(E_{2 i, 2 i}\right)^{\perp} \varphi\left(E_{2 i-1,2 i}\right) \varphi\left(E_{2 i, 2 i}\right) \\
+\varphi\left(E_{2 i, 2 i}\right) \varphi\left(E_{2 i-1,2 i}\right) \varphi\left(E_{2 i, 2 i}\right)^{\perp}=\varphi\left(E_{2 i-1,2 i}\right)
\end{aligned}
$$

and

$$
\begin{aligned}
& \varphi\left(E_{2 i-1,2 i-1}\right) \varphi\left(E_{2 i-1,2 i}\right) \varphi\left(E_{2 i-1,2 i-1}\right)^{\perp} \\
& +\varphi\left(E_{2 i-1,2 i-1}\right)^{\perp} \varphi\left(E_{2 i-1,2 i}\right) \varphi\left(E_{2 i-1,2 i-1}\right)=\varphi\left(E_{2 i-1,2 i}\right) .
\end{aligned}
$$

Then

$$
E_{k k}^{\perp} \varphi\left(E_{2 i-1,2 i}\right) E_{k k}+E_{k k} \varphi\left(E_{2 i-1,2 i}\right) E_{k k}^{\perp}=\varphi\left(E_{2 i-1,2 i}\right)
$$




$$
\begin{aligned}
& \text { and } \\
& E_{j j} \varphi\left(E_{2 i-1,2 i}\right) E_{j j}^{\perp}+E_{j j}^{\perp} \varphi\left(E_{2 i-1,2 i}\right) E_{j j}=\varphi\left(E_{2 i-1,2 i}\right) .
\end{aligned}
$$

So we can get the following from the second equation of $(*)$;

(1) If $j$ is 1 , then $\varphi\left(E_{2 i-1,2 i}\right)$ is a matrix all of whose entries are zero except for the $(1,2)$-component and the $(1,2 n)$-component.

(2) If $j$ is an odd number and $j \neq 1$, then $\varphi\left(E_{2 i-1,2 i}\right)$ is a matrix all of whose entries are zero except for the $(j, j-1)$-component and the $(j, j+1)$-component.

(3) If $j$ is 2 , then $\varphi\left(E_{2 i-1,2 i}\right)$ is a matrix all of whose entries are zero except for the $(1,2)$-component and the $(3,2)$-component.

(4) If $j$ is an even number and $j \neq 2$, then $\varphi\left(E_{2 i-1,2 i}\right)$ is a matrix all of whose entries are zero except for the $(j-1, j)$-component and the $(j+1, j)$-component.

$(\alpha)$ From the first equation of $(*)$ we know the following: If $k$ is 1 , then $\varphi\left(E_{2 i-1,2 i}\right)$ is a matrix all of whose entries are zero except for the $(1,2)$-component.

$(\beta)$ If $k$ is an odd number and $k \neq 1$, then $\varphi\left(E_{2 i-1,2 i}\right)$ is a matrix all of whose entries are zero except for the $(k, k-1)$-component and the $(k, k+1)$-component.

( $\tau)$ If $k$ is 2 , then $\varphi\left(E_{2 i-1,2 i}\right)$ is a matrix all of whose entries are zero except for the $(1,2)$-component and the $(3,2)$-component.

$(\delta)$ If $k$ is an even number and $k \neq 2$, then $\varphi\left(E_{2 i-1,2 i}\right)$ is a matrix all of whose entries are zero except for the $(k-1, k)$-component and the $(k+1, k)$-component.

Then the following cannot happen at the same time;

(1) and $(\alpha)$ because $j \neq k$.

(1) and $(\beta)$ because $j=1$ and $k \geq 3$.

(1) and $(\delta)$ because $k>2$.

(2) and $(\alpha)$ because $j \neq 1$.

(2) and $(\beta)$ because $j \neq k$.

(3) and $(\tau)$ because $j \neq k$.

(3) and $(\delta)$ because $k>2$.

(4) and $(\alpha)$ because $j>2$.

(4) and $(\tau)$ because $j>2$.

(4) and $(\delta)$ because $j \neq k$.

Then the following can happen at the same time;

(1) and $(\tau)$ if $|k-j|=1$.

(2) and $(\tau)$ if $j=3$ and so $|j-k|=1$.

(2) and $(\delta)$ if $|j-k|=1$. 
(3) and $(\alpha)$ if $|j-k|=1$.

(3) and $(\beta)$ if $k=3$ and so $|j-k|=1$.

(4) and $(\tau)$ if $|j-k|=1$.

So we can get the result of the theorem.

Note that in all cases, $\varphi\left(E_{2 i-1,2 i}\right)$ is a scalar multiple of $E_{k j}$ or $E_{j k}$. From this theorem, we can get the following corollary.

CoRollary 17. Let $\varphi: \operatorname{Alg} \mathscr{L}_{\infty} \rightarrow \operatorname{Alg} \mathscr{L}_{\infty}$ be a surjective isometry such that $\varphi(I)=I$. Then $(1) \varphi\left(E_{i i}\right)=E_{i i}$ for all $i ; i=1,2,3, \ldots$ and (2) $\varphi\left(\mathscr{L}_{\infty}\right)=\mathscr{L}_{\infty}$.

Proof. Suppose that $\varphi\left(E_{11}\right)=E_{i i}$ for $i \neq 1$. Then $\varphi\left(E_{22}\right)=E_{i-1, i-1}$ or $\varphi\left(E_{22}\right)=E_{i+1, i+1}$ by Theorem 16. If $\varphi\left(E_{22}\right)=E_{i-1, i-1}$, then $\varphi\left(E_{33}\right)=E_{i-2, i-2}$, and by continuing we get $\varphi\left(E_{i i}\right)=E_{11}$. Let $\varphi\left(E_{i+1, i+1}\right)=E_{k k}$. Then since $k \geq i+1, k-1 \neq 1$, contradicting Theorem 16. If $\varphi\left(E_{22}\right)=E_{i+1, i+1}$, then by Theorem $16 \varphi\left(E_{33}\right)=$ $E_{i+2, i+2}, \ldots, \varphi\left(E_{k k}\right)=E_{i+k-1, i+k-1}, \cdots(*)$. But since $\varphi$ is a surjective isometry, $\varphi\left(E_{j j}\right)=E_{11}$ for some $j$. But $\varphi\left(E_{j j}\right)=E_{i+j-1, i+j-1}$ by $(*)$. Then $i+j-1=1$. So $j=2-i$, which is impossible because $i \geq 2$. Thus $\varphi\left(E_{11}\right)=E_{11}$ and hence $\varphi\left(E_{i i}\right)=E_{i i}$ for all $i$ by Theorem 16 . By (1) $\varphi\left(\mathscr{L}_{\infty}\right)=\mathscr{L}_{\infty}$.

LEMMA 18. Let $\varphi: \operatorname{Alg} \mathscr{L}_{2 n} \rightarrow \operatorname{Alg} \mathscr{L}_{2 n}$ be a surjective isometry such that $\varphi(I)=I$. Let $\varphi\left(E_{11}\right)=E_{i i}$ and let $\varphi\left(E_{22}\right)=E_{k k}$. If $1<i<2 n$, then $|i-k|=1$.

Proof. Since $E_{11} E_{12} E_{11}^{\perp}=E_{12}$ and $E_{22}^{\perp} E_{12} E_{22}=E_{12}, E_{i i} \varphi\left(E_{12}\right) E_{i i}^{\perp}+$ $E_{i i}^{\perp} \varphi\left(E_{12}\right) E_{i i}=\varphi\left(E_{12}\right)$ and $E_{k k}^{\perp} \varphi\left(E_{12}\right) E_{k k}+E_{k k} \varphi\left(E_{12}\right) E_{k k}^{\perp}=\varphi\left(E_{12}\right)$.

(1) If $i$ is an odd number, then $\varphi\left(E_{12}\right)$ is a $2 n \times 2 n$ matrix whose entries are zero except for the $(i, i-1)$-component and the $(i, i+1)$ component.

(2) If $i$ is an even number, then $\varphi\left(E_{12}\right)$ is a $2 n \times 2 n$ matrix whose entries are zero except for the $(i-1, i)$-component and the $(i+1, i)$ component.

$(\alpha)$ If $k$ is an odd number, the $\varphi\left(E_{12}\right)$ is a $2 n \times 2 n$ matrix whose entries are zero except for the $(k, k-1)$-component and the $(k, k+1)$ component.

$(\beta)$ If $k$ is an even number, then $\varphi\left(E_{12}\right)$ is a $2 n \times 2 n$ matrix whose entries are zero except for the $(k-1, k)$-component and the $(k+1, k)$ component.

Then the following combinations are impossible; 
(1) and $(\alpha)$ because $i \neq k$.
(2) and $(\beta)$ because $i \neq k$.

The following combinations are possible;

(1) and $(\beta)$ if $|i-k|=1$.

(2) and $(\alpha)$ if $|i-k|=1$.

By an argument similar to Lemma 18, we can get the following lemma.

LEMMA 19. Let $\varphi: \operatorname{Alg} \mathscr{L}_{2 n} \rightarrow \operatorname{Alg} \mathscr{L}_{2 n}$ be a surjective isometry such that $\varphi(I)=I$. Let $\varphi\left(E_{2 i-1,2 i-1}\right)=E_{j j}$ and let $\varphi\left(E_{2 i, 2 i}\right)=E_{k k}$. If $1<j<2 n$, then $|j-k|=1$.

From Lemma 18 and Lemma 19, we can get the following corollary.

Corollary 20. Let $\varphi: \operatorname{Alg} \mathscr{L}_{2 n} \rightarrow \operatorname{Alg} \mathscr{L}_{2 n}\left(\right.$ or $\left.\operatorname{Alg} \mathscr{L}_{\infty} \rightarrow \operatorname{Alg} \mathscr{L}_{\infty}\right)$ be a surjective isometry such that $\varphi(I)=I, \varphi\left(E_{2 i-1,2 i-1}\right)=E_{j j}$ and $\varphi\left(E_{2 i, 2 i}\right)=E_{k k}$. If $1<j<2 n$, then $\varphi\left(E_{2 i-1,2 i-2}\right)$ and $\varphi\left(E_{2 i-1,2 i}\right)$ have the form

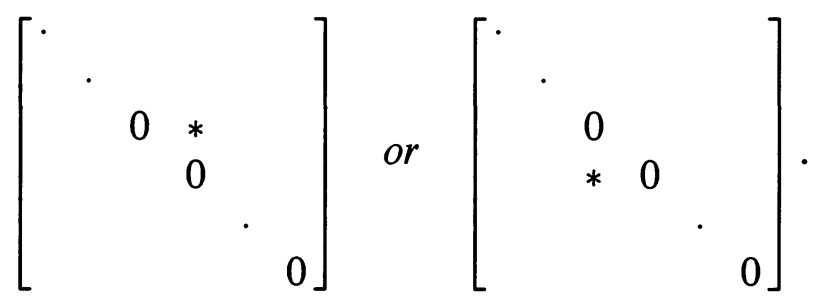

In particular, if $\varphi\left(E_{i i}\right)=E_{i i}$ for each $i(i=1,2, \ldots, 2 n)$, then there exists a complex number $\alpha_{i j}$ such that $\varphi\left(E_{i j}\right)=\alpha_{i j} E_{i j}$ for each $E_{i j}$ in $\operatorname{Alg} \mathscr{L}_{2 n}$ (or $E_{i j}$ in $\operatorname{Alg} \mathscr{L}_{\infty}$ ).

In the following, we will investigate $\varphi\left(\mathscr{L}_{2 n}\right)$ case by case.

LEMMA 21. If $\varphi: \operatorname{Alg} \mathscr{L}_{2 n} \rightarrow \operatorname{Alg} \mathscr{L}_{2 n}$ is a surjective isometry such that $\varphi(I)=I$ and if $\varphi\left(E_{11}\right)=E_{11}$, then $\varphi\left(\mathscr{L}_{2 n}\right)=\mathscr{L}_{2 n}$.

Proof. Since $E_{11} E_{12} E_{11}^{\perp}=E_{12}, E_{11} \varphi\left(E_{12}\right) E_{11}^{\perp}+E_{11}^{\perp} \varphi\left(E_{12}\right) E_{11}=$ $\varphi\left(E_{12}\right)$. So $\varphi\left(E_{12}\right)$ is a $2 n \times 2 n$ matrix whose entries are zero except for the $(1,2)$-component and the $(1,2 n)$-component. Set $\varphi\left(E_{22}\right)=E_{k k}$. Since $E_{22}^{\perp} E_{12} E_{22}=E_{12}, E_{k k}^{\perp} \varphi\left(E_{12}\right) E_{k k}+E_{k k} \varphi\left(E_{12}\right) E_{k k}^{\perp}=\varphi\left(E_{12}\right)$. So the only possibility is $k=2$ or $k=2 n$. Assume that $k=2$. Then $\varphi\left(E_{i i}\right)=E_{i i}$ for all $i$ by Lemma $19 ; i=1,2, \ldots, 2 n$. In this case, $\varphi\left(\mathscr{L}_{2 n}\right)=\mathscr{L}_{2 n}$. Assume that $k=2 n$. Since $E_{22}^{\perp} E_{32} E_{22}=E_{32}$ and $E_{33} E_{32} E_{33}^{\perp}=E_{32}, E_{2 n, 2 n}^{\perp} \varphi\left(E_{32}\right) E_{2 n, 2 n}+E_{2 n, 2 n} \varphi\left(E_{32}\right) E_{2 n, 2 n}^{\perp}=\varphi\left(E_{32}\right)$ 
and $E_{j j}^{\perp} \varphi\left(E_{32}\right) E_{j j}+E_{j j} \varphi\left(E_{32}\right) E_{j j}^{\perp}=\varphi\left(E_{32}\right)$, where $E_{j j}=\varphi\left(E_{33}\right)$. We know that $j \neq 1$ and $j \neq 2 n$. By the first equation, $\varphi\left(E_{32}\right)$ is a $2 n \times 2 n$ matrix whose entries are zero except for the $(1,2 n)$-component and the $(2 n-1,2 n)$-component. If $j$ is an odd number, then $\varphi\left(E_{32}\right)$ is a $2 n \times 2 n$ matrix whose entries are zero except for the $(j, j-1)$ component and the $(j, j+1)$-component. If $j$ is an even number, then $\varphi\left(E_{32}\right)$ is a $2 n \times 2 n$ matrix whose entries are zero except for the $(j-1, j)$-component and the $(j+1, j)$-component. So the only possibility is $j=2 n-1$, that is, $\varphi\left(E_{33}\right)=E_{2 n-1,2 n-1}$. By Lemma 19, $\varphi\left(E_{44}\right)=E_{2 n-2,2 n-2}, \ldots, \varphi\left(E_{2 n, 2 n}\right)=E_{22}$. In this case, if $\varphi\left(E_{k k}\right)=$ $E_{j j}$, then $k$ and $j$ have the same parity and it is straightforward to see that $\varphi\left(\mathscr{L}_{2 n}\right)=\mathscr{L}_{2 n}$.

CoRollary 22. If $\varphi: \operatorname{Alg} \mathscr{L}_{2 n} \rightarrow \operatorname{Alg} \mathscr{L}_{2 n}$ is a surjective isometry such that $\varphi(I)=I$ and $\varphi\left(E_{11}\right)=E_{2 n, 2 n}$, then $\varphi\left(\mathscr{L}_{2 n}\right)=\mathscr{L}_{2 n}^{\perp}$.

Proof. Let $\varphi_{1}: \operatorname{Alg} \mathscr{L}_{2 n} \rightarrow \operatorname{Alg} \mathscr{L}_{2 n}$ be the surjective isometry in Example 4. Then $\varphi_{1} \circ \varphi: \operatorname{Alg} \mathscr{L}_{2 n} \rightarrow \operatorname{Alg} \mathscr{L}_{2 n}$ is a surjective isometry such that $\varphi_{1} \circ \varphi(I)=I$ and $\varphi_{1} \circ \varphi\left(E_{11}\right)=\varphi_{1}\left(E_{2 n, 2 n}\right)=E_{11}$. So $\varphi_{1} \circ \varphi\left(\mathscr{L}_{2 n}\right)=\mathscr{L}_{2 n}$ by Lemma 21 . Since $\varphi_{1}\left(\mathscr{L}_{2 n}\right)=\mathscr{L}_{2 n}^{\perp}, \varphi\left(\mathscr{L}_{2 n}\right)=\mathscr{L}_{2 n}^{\perp}$.

LEMMA 23. Let $\varphi: \operatorname{Alg} \mathscr{L}_{2 n} \rightarrow \operatorname{Alg} \mathscr{L}_{2 n}$ be a surjective isometry such that $\varphi(I)=I$ and $\varphi\left(\mathscr{L}_{2 n}\right)=\mathscr{L}_{2 n}^{\perp}$. Then $\varphi\left(\mathscr{L}_{2 n}^{\perp}\right)=\mathscr{L}_{2 n}$.

Proof. $\varphi\left(\mathscr{L}_{2 n}^{\perp}\right)=\varphi\left(\mathscr{L}_{2 n}\right)^{\perp}=\left(\mathscr{L}_{2 n}^{\perp}\right)^{\perp}=\mathscr{L}_{2 n}$.

CoRollaRY 24. Let $\varphi: \operatorname{Alg} \mathscr{L}_{2 n} \rightarrow \operatorname{Alg} \mathscr{L}_{2 n}$ be a surjective isometry such that $\varphi(I)=I$. Let $\varphi\left(E_{11}\right)=E_{i i} ; i \neq 1$ and $i \neq 2 n$. If $i$ is an odd number, then $\varphi\left(\mathscr{L}_{2 n}\right)=\mathscr{L}_{2 n}$. If $i$ is an even number, then $\varphi\left(\mathscr{L}_{2 n}\right)=\mathscr{L}_{2 n}^{\perp}$.

Proof. First, let $i=2 k-1$, for some $k$. Let $\varphi_{1}$ be the surjective isometry in Example 2; that is, $\varphi_{1}\left(E_{11}\right)=E_{2 k-1,2 k-1}$. Then $\varphi_{1} \circ$ $\varphi\left(E_{11}\right)=\varphi_{1}\left(E_{2 k-1,2 k-1}\right)=E_{11}$. By Lemma $21, \varphi_{1} \circ \varphi\left(\mathscr{L}_{2 n}\right)=\mathscr{L}_{2 n}$. So $\varphi\left(\mathscr{L}_{2 n}\right)=\varphi_{1}^{-1}\left(\mathscr{L}_{2 n}\right)$. Since $\varphi_{1}\left(\mathscr{L}_{2 n}\right)=\mathscr{L}_{2 n}, \varphi\left(\mathscr{L}_{2 n}\right)=\varphi_{1}^{-1}\left(\mathscr{L}_{2 n}\right)=$ $\mathscr{L}_{2 n}$. Let $i=2 k$ for some $k$. Let us consider $V_{2 n-2 k+1}$ in Example 3 and let $\varphi_{2}: \operatorname{Alg} \mathscr{L}_{2 n} \rightarrow \operatorname{Alg} \mathscr{L}_{2 n}$ be a surjective isometry in Example 3. Then $\varphi_{2} \circ \varphi: \operatorname{Alg} \mathscr{L}_{2 n} \rightarrow \operatorname{Alg} \mathscr{L}_{2 n}$ is a surjective isometry such that $\varphi_{2} \circ \varphi(I)=I$ and $\varphi_{2} \circ \varphi\left(E_{11}\right)=\varphi_{2}\left(E_{2 k, 2 k}\right)=E_{2 n, 2 n}$. By Corollary $22, \varphi_{2} \circ \varphi\left(\mathscr{L}_{2 n}\right)=\mathscr{L}_{2 n}^{\perp}$. So $\varphi\left(\mathscr{L}_{2 n}\right)=\varphi_{2}^{-1}\left(\mathscr{L}_{2 n}^{\perp}\right)$. Since $\varphi_{2}\left(\mathscr{L}_{2 n}\right)=\mathscr{L}_{2 n}$, $\varphi\left(\mathscr{L}_{2 n}\right)=\mathscr{L}_{2 n}^{\perp}$. 
If we summarize lemmas and corollaries, then we can get the following theorem.

THEOREM 25. Let $\varphi: \operatorname{Alg} \mathscr{L}_{2 n} \rightarrow \operatorname{Alg} \mathscr{L}_{2 n}$ be a surjective isometry such that $\varphi(I)=I$. Let $\varphi\left(E_{11}\right)=E_{i i}$. If $i$ is an odd number, then $\varphi\left(\mathscr{L}_{2 n}\right)=\mathscr{L}_{2 n}$. If $i$ is an even number, then $\varphi\left(\mathscr{L}_{2 n}\right)=\mathscr{L}_{2 n}$.

Let $\varphi: \operatorname{Alg} \mathscr{L}_{2 n} \rightarrow \operatorname{Alg} \mathscr{L}_{2 n}$ be a surjective isometry such that $\varphi(I)=I$ and $\varphi\left(\mathscr{L}_{2 n}\right)=\mathscr{L}_{2 n}^{\perp}$. If $J$ is the bijective conjugation which is defined below, then for all $x, y$ in $\mathbf{C}^{2 n}$ and all $\alpha$ in $\mathbf{C}$

(1) $J(x+y)=J x+J y$,

(2) $J(\alpha x)=\bar{\alpha} J x$

(3) $(J x, J y)=(y, x)$,

(4) $(J x, y)=(J y, x)$ and

(5) $J^{2}=I$.

\section{Define}

$$
J\left(x_{1}, x_{2}, \ldots, x_{2 n}\right)^{t}=\left(\bar{x}_{2 n}, \bar{x}_{2 n-1}, \ldots, \bar{x}_{1}\right)^{t}
$$

for every $\left(x_{1}, x_{2}, \ldots, x_{2 n}\right)^{t}$ in $\mathbf{C}^{2 n}$.

If $A$ is in $\operatorname{Alg} \mathscr{L}_{2 n}$, then the map $A \rightarrow J A^{*} J$ is linear and "flips" $A$ across the northeast-southwest diagonal (see Example 4).

Define $\varphi_{1}: \operatorname{Alg} \mathscr{L}_{2 n} \rightarrow \operatorname{Alg} \mathscr{L}_{2 n}$ by $\varphi_{1}(A)=J A^{*} J$ for every $A$ in $\operatorname{Alg} \mathscr{L}_{2 n}$. Then $\varphi_{1}$ is well-defined by the above statement, linear, $\varphi_{1}$ is a surjective isometry, and $\varphi_{1}\left(\mathscr{L}_{2 n}\right)=\mathscr{L}_{2 n}^{\perp}$. If $\varphi\left(\mathscr{L}_{2 n}\right)=\mathscr{L}_{2 n}^{\perp}$, then define $\tilde{\tilde{\varphi}}=\varphi_{1} \circ \varphi: \operatorname{Alg} \mathscr{L}_{2 n} \rightarrow \operatorname{Alg} \mathscr{L}_{2 n}$. Then $\tilde{\tilde{\varphi}}\left(\mathscr{L}_{2 n}\right)=\varphi_{1} \circ \varphi\left(\mathscr{L}_{2 n}\right)=$ $\varphi_{1}\left(\mathscr{L}_{2 n}^{\perp}\right)=\mathscr{L}_{2 n}$ by Lemma 23 .

Since $(J A J)^{*}=J A^{*} J, \varphi_{1}^{-1}=\varphi_{1}$ and we can get the following theorem.

THEOREM 26. Let $\varphi: \operatorname{Alg} \mathscr{L}_{2 n} \rightarrow \operatorname{Alg} \mathscr{L}_{2 n}$ be a surjective isometry such that $\varphi(I)=I$ and $\varphi\left(\mathscr{L}_{2 n}\right)=\mathscr{L}_{2 n}^{\perp}$. Then, there exist unitary operators $U$ and $V$ such that $\tilde{\tilde{\varphi}}(A)=U A V$ if and only if $\varphi(A)=J V^{*} A^{*} U^{*} J$ for every $A$ in $\operatorname{Alg} \mathscr{L}_{2 n}$.

Let $\varphi: \operatorname{Alg} \mathscr{L}_{\infty} \rightarrow \operatorname{Alg} \mathscr{L}_{\infty}$ be a surjective isometry such that $\varphi(I)=$ $I, \varphi\left(E_{i i}\right)=E_{i i}$ for each $i ; i=1,2, \ldots$ and $\varphi\left(\mathscr{L}_{\infty}\right)=\mathscr{L}_{\infty}$. Then by Corollary 20, there exists $\alpha_{i j}$ in $\mathbf{C}$ such that $\varphi\left(E_{i j}\right)=\alpha_{i j} E_{i j}$ for all $E_{i j}$ in $\operatorname{Alg} \mathscr{L}_{\infty}(|i-j|=1)$. Then we claim that there exists a diagonal unitary $U$ such that $\varphi\left(E_{i j}\right)=U E_{i j} U^{*}$ for all $E_{i j}$ in $\operatorname{Alg} \mathscr{L}_{\infty}(|i-j|=1)$. Let $U$ be a diagonal matrix whose $(j, j)$-component is $e^{i \theta_{j}}$ for all $j$ $(j=1,2, \ldots)$. 
Then the equation $\varphi\left(E_{i j}\right)=U E_{i j} U^{*}$ holds for all $E_{i j}$ in $\operatorname{Alg} \mathscr{L}_{\infty}$ provided the following system can be solved

$$
\begin{aligned}
& e^{i\left(\theta_{1}-\theta_{2}\right)}=\alpha_{12} . \\
& e^{i\left(\theta_{3}-\theta_{2}\right)}=\alpha_{32} . \\
& e^{i\left(\theta_{3}-\theta_{4}\right)}=\alpha_{34} .
\end{aligned}
$$

The equation can be solved recursively ( $\theta_{1}$ may be set equal to 0$)$. From these facts, we can get the following theorem.

TheOREM 27. If $\varphi: \operatorname{Alg} \mathscr{L}_{\infty} \rightarrow \operatorname{Alg} \mathscr{L}_{\infty}$ is a surjective isometry such that $\varphi(I)=I, \varphi\left(E_{i i}\right)=E_{i i}$ for all $i(i=1,2, \ldots)$ and $\varphi\left(\mathscr{L}_{\infty}\right)=\mathscr{L}_{\infty}$, then there exists a diagonal unitary operator $U$ whose $(j, j)$-component is $e^{i \theta_{j}}$ for all $j(j=1,2, \ldots)$ such that $\varphi(A)=U A U^{*}$ for every $A$ in $\operatorname{Alg} \mathscr{L}_{\infty}$.

For the rest we will consider a surjective isometry such that $\varphi\left(\mathscr{L}_{2 n}\right)$ $=\mathscr{L}_{2 n}$. As a special case, we first consider $n=1$.

THEOREM 28. Let $\varphi: \operatorname{Alg} \mathscr{L}_{2} \rightarrow \operatorname{Alg} \mathscr{L}_{2}$ be a surjective isometry such that $\varphi(I)=I$ and $\varphi\left(E_{i i}\right)=E_{i i} ; i=1,2$. Then there exists a unitary operator $U$ such that $\varphi(A)=U A U^{*}$ for every $A$ in $\operatorname{Alg} \mathscr{L}_{2}$.

Proof. Let

$$
U=\left[\begin{array}{cc}
e^{i \theta_{1}} & 0 \\
0 & e^{i \theta_{2}}
\end{array}\right]
$$

Let

$$
A=\left[\begin{array}{cc}
a_{11} & a_{12} \\
0 & a_{22}
\end{array}\right] \text { and } \varphi(A)=\left[\begin{array}{cc}
a_{11} & b_{12} \\
0 & a_{22}
\end{array}\right] \text {. }
$$

Then there exists a complex number $\alpha$ such that $a_{12}=\alpha b_{12}$. This $\alpha$ depends only on $\varphi$ (by linearity), not on the matrix entries. Note that $|\alpha|=1$ because $\varphi$ is an isometry. If we fix $e^{i \theta_{1}}$ and if we determine $e^{i \theta_{2}}$ such that $e^{i \theta_{1}} e^{-i \theta_{2}}=\alpha$, then $\varphi(A)=U A U^{*}$ for every $A$ in $\operatorname{Alg} \mathscr{L}_{2}$.

LEMMA 29. Let $U$ be a unitary operator. Then $\|I+U\|=2$ if and only if 1 is in $\sigma(U)$.

Proposition 30. Let $A$ be an $n \times n$ matrix $(n \geq 2)$ with 1 on the diagonal and just below it, 1 the $(1, n)$-component and 0 elsewhere. Then $\|A\|=2$.

Proof. Let $U$ be an $n \times n$ matrix with 1 just below the diagonal, 1 the $(1, n)$-component and 0 elsewhere. Since $U\left(x_{1}, x_{2}, \ldots, x_{n}\right)^{t}=$ 
$\left(x_{n}, x_{1}, \ldots, x_{n-1}\right)^{t}$ for every vector $\left(x_{1}, x_{2}, \ldots, x_{n}\right)^{t}$ in $\mathbf{C}^{n}, U$ is a unitary operator. Then $A=I+U$. Let $X$ be a vector in $\mathbf{C}^{n}$ all of whose entries are 1. Then since $U X=X, 1$ is in $\sigma(U)$. So $\|A\|=2$ by Lemma 29.

Proposition 31. Let $U$ be an $n \times n$ matrix with $t_{i}$ the $(i+1, i)$ component and $t_{n}$ the $(1, n)$-component $(i=1,2, \ldots, n-1)$. If 1 is in $\sigma(U)$ and $\left|t_{i}\right|=1$ for every $i ; i=1,2, \ldots, n$, then $U$ is a unitary operator and $\prod_{i=1}^{n} t_{i}=1$.

Proof. Since $U\left(x_{1}, x_{2}, \ldots, x_{n}\right)^{t}=\left(t_{n} x_{n}, t_{1} x_{1}, t_{2} x_{2}, \ldots, t_{n-1} x_{n-1}\right)^{t}$ for every vector $\left(x_{1}, x_{2}, \ldots, x_{n}\right)^{t}$ in $\mathbf{C}^{n}, U$ is a unitary operator. Since 1 is in $\sigma(U)$, there exists a non zero vector $\left(x_{1}, x_{2}, \ldots, x_{n}\right)^{t}$ such that

$$
\begin{aligned}
U\left(x_{1}, x_{2}, \ldots, x_{n}\right)^{t} & =\left(t_{n} x_{n}, t_{1} x_{1}, t_{2} x_{2}, \ldots, t_{n-1} x_{n-1}\right)^{t} \\
& =\left(x_{1}, x_{2}, \ldots, x_{n}\right)^{t} .
\end{aligned}
$$

So $t_{n} x_{n}=x_{1}, t_{1} x_{1}=x_{2}, t_{2} x_{2}=x_{3}, \ldots, t_{n-1} x_{n-1}=x_{n}$. If $x_{i}=0$ for some $i(1 \leq i \leq n)$, then $x_{1}=x_{2}=\cdots=x_{n}=0$. So $x_{i} \neq 0$ for every $i$ $(i=1,2, \ldots, n)$. Then $\left(\prod_{i=1}^{n} t_{i}\right) \prod_{i=1}^{n} x_{i}=\prod_{i=1}^{n} x_{i}$. Hence, $\prod_{i=1}^{n} t_{i}=1$.

Proposition 32. Let $A$ be an $n \times n$ matrix with $a_{i}$ the $(i, i)$-component $(i=1,2, \ldots, n), s_{j}$ the $(j+1, j)$-component $(j=1,2, \ldots, n-1), s_{n}$ the $(1, n)$-component and 0 elsewhere. If $\left|a_{i}\right|=\left|s_{i}\right|=1(i=1,2, \ldots, n)$ and $\|A\|=2$, then $\prod_{i=1}^{n} a_{i}=\prod_{i=1}^{n} s_{i}$.

Proof. Let $U$ be an $n \times n$ diagonal matrix whose ( $i, i)$-component is $a_{i}^{-1}$ for all $i(i=1,2, \ldots, n)$. Then $U A$ is the $n \times n$ matrix with 1 on the diagonal, $a_{i+1}^{-1} s_{i}$ the $(i+1, i)$-component $(i=1,2, \ldots, n-1)$, $a_{1}^{-1} s_{n}$ the $(1, n)$-component and 0 elsewhere. Let $V$ be an $n \times n$ matrix with $a_{i+1}^{-1} s_{i}$ the $(i+1,1)$-component $(i=1,2, \ldots, n-1), a_{1}^{-1} s_{n}$ the $(1, n)$-component and 0 elsewhere. Then $V$ is a unitary operator and $U A=I+V$. Since $U$ is a unitary operator, $\|U A\|=\|A\|=\|I+V\|=2$. By Lemma 29, 1 is in $\sigma(V)$. Since

$$
\left|a_{1}^{-1} s_{n}\right|=\left|a_{2}^{-2} s_{1}\right|=\left|a_{3}^{-1} s_{2}\right|=\cdots=\left|a_{n}^{-1} s_{n-1}\right|=1,
$$

by Proposition 31,

$$
\left(\prod_{i=1}^{n}\left(a_{i+1}\right)^{-1} s_{i}\right) a_{1}^{-1} s_{n}=\left(\prod_{i=1}^{n} a_{i}^{-1}\right)\left(\prod_{i=1}^{n} s_{i}\right)=1 .
$$

Hence $\prod_{i=1}^{n} a_{i}=\prod_{i=1}^{n} s_{i}$. 
LEMMA 33. Let $\varphi: \operatorname{Alg} \mathscr{L}_{2 n} \rightarrow \operatorname{Alg} \mathscr{L}_{2 n}$ be a surjective isometry such that $\varphi\left(E_{i i}\right)=E_{i i}$ for each $i ; i=1,2, \ldots, 2 n$ and $n \geq 2$. Let $\varphi\left(E_{i j}\right)=$ $\alpha_{i j} E_{i j}$ for all $E_{i j}$ in $\operatorname{Alg} \mathscr{L}_{2 n}$, where $\left|\alpha_{i j}\right|=1$ for all $i, j$. Then

$$
\alpha_{12} \bar{\alpha}_{32} \alpha_{34} \bar{\alpha}_{54} \alpha_{56} \cdots \alpha_{2 n-1,2 n} \bar{\alpha}_{1,2 n}=1 \text {. }
$$

Proof. Let $A$ be a $2 n \times 2 n$ matrix with 1 the $(2 i-1,2 i)$-component $(i=1,2, \ldots, n)$ and the $(2 j+1,2 j)$-component $(j=1,2, \ldots, n-1)$ and the $(1,2 n)$-component, and 0 elsewhere. Then, by hypothesis, $\varphi(A)=\left(\alpha_{i j}\right)$. Let $B$ be the $n \times n$ matrix with 1 on the diagonal and just below it, 1 the $(1, n)$-component and 0 elsewhere. Note that the $n \times n$ matrix $B$ and the $2 n \times 2 n$ matrix $A$ have the same norm. Let $D$ be the $n \times n$ matrix with $\alpha_{2 i-1,2 i}$ the $(i, i)$-component $(i=1,2, \ldots, n), \alpha_{1,2 n}$ the $(1, n)$-component, $\alpha_{2 j+1,2 j}$ the $(j+1, j)$-component $(j=1,2, \ldots$, $n-1)$ and 0 elsewhere. Then $\|D\|=\|\varphi(A)\|$. Since $\varphi$ preserves norm, $\|A\|=\|\varphi(A)\|$. So $\|B\|=\|D\|$. By Proposition $30\|B\|=2$ and hence $\|D\|=2$. Since $\left|\alpha_{2 i-1,2 i}\right|=\left|\alpha_{2 i-1,2 i-2}\right|=1$ for each $i ; i=1,2, \ldots, n$.

$$
\alpha_{12} \bar{\alpha}_{32} \alpha_{34} \bar{\alpha}_{54} \alpha_{56} \cdots \bar{\alpha}_{2 n-1,2 n-2} \alpha_{2 n-1,2 n} \bar{\alpha}_{1,2 n}=1
$$

by Proposition 32 .

THEOREM 34. Let $\varphi: \operatorname{Alg} \mathscr{L}_{2 n} \rightarrow \operatorname{Alg} \mathscr{L}_{2 n}$ be a surjective isometry such that $\varphi\left(E_{i i}\right)=E_{i i}$ for each $i ; i=1,2, \ldots, 2 n$ and $n \geq 2$. Then there exists a unitary operator $V$ such that $\varphi(A)=V A V^{*}$ for every $A$ in $\operatorname{Alg} \mathscr{L}_{2 n}$.

Proof. Let $A=\left(a_{i j}\right)$ be in $\operatorname{Alg} \mathscr{L}_{2 n}$ and let $\varphi\left(E_{i j}\right)=\alpha_{i j} E_{i j}$ for all $E_{i j}$ in $\operatorname{Alg} \mathscr{L}_{2 n}$, where $\left|\alpha_{i j}\right|=1$ for all $\alpha_{i j}$.

Let $V$ be a $2 n \times 2 n$ diagonal matrix whose $(j, j)$-component is $e^{i \theta_{j}}$ for all $j(j=1,2, \ldots, 2 n)$. Then $V A V^{*}$ is the $2 n \times 2 n$ matrix with $a_{r r}$ the $(r, r)$-component $(r=1,2, \ldots, 2 n), e^{i \theta_{p}} a_{p, p+1} e^{-\theta_{p+1}}$ the $(p, p+1)$-component $(p=1,3, \ldots, 2 n-1), e^{i \theta_{q}} a_{q, q-1} e^{-i \theta_{q-1}}$ the $(q, q-1)$-component $(q=3,5, \ldots, 2 n-1), e^{i \theta_{1}} a_{1,2 n} e^{-i \theta_{2 n-1}}$ the $(1,2 n)$ component and 0 elsewhere.

So the theorem will be proved if we can determine $e^{i \theta_{1}}, e^{i \theta_{2}}, \ldots, e^{i \theta_{2^{n}}}$ satisfying the following relations;

$$
\begin{aligned}
e^{i \theta_{1}} e^{-i \theta_{2}} & =\alpha_{12} . \\
e^{i \theta_{3}} e^{-i \theta_{2}} & =\alpha_{32} . \\
e^{i \theta_{3}} e^{-i \theta_{4}} & =\alpha_{34} . \\
\vdots & \\
e^{i \theta_{2 n-1}} e^{-i \theta_{2 n}} & =\alpha_{2 n-1,2 n} . \\
e^{i \theta_{1}} e^{-i \theta_{2 n}} & =\alpha_{1,2 n} .
\end{aligned}
$$


Let $\alpha_{i j}=e^{i \theta}$ for all $i, j$ such that $E_{i j}$ is in $\operatorname{Alg} \mathscr{L}_{2 n}$. Then $\theta_{12}, \theta_{32}$, $\theta_{34}, \ldots, \theta_{2 n-1,2 n}$ and $\theta_{1,2 n}$ are known by $\alpha_{12}, \alpha_{32}, \alpha_{34}, \ldots, \alpha_{2 n-1,2 n}$ and $\alpha_{1,2 n}$ respectively. It will suffice to solve the linear system; $(*) \ldots, \theta_{1}-\theta_{2}=\theta_{12}, \theta_{3}-\theta_{2}=\theta_{32}, \ldots, \theta_{2 n-1}-\theta_{2 n}=\theta_{2 n-1,2 n}$ and $\theta_{1}-\theta_{2 n}=\theta_{1,2 n}$.

Let $A$ be the matrix of coefficients of $(*)$ and let $A^{1}, A^{2}, \ldots, A^{2 n}$ be the column vectors of $A$. Let $B=\left(\theta_{12}, \theta_{32}, \theta_{34}, \ldots, \theta_{2 n-1,2 n}, \theta_{1,2 n}\right)^{t}$. Then the system $(*)$ has solutions if and only if $\operatorname{rank} A=$ $\operatorname{rank}\left(A^{1}, A^{2}, A^{3}, \ldots, A^{2 n}, B\right)$.

It is easy to check that the left hand side is $n-1$. Thus, the rank of the right hand side must be $n-1$ and the ranks will be equal if

$$
\theta_{12}-\theta_{32}+\theta_{34}-\cdots+\theta_{2 n-1,2 n}-\theta_{1,2 n}=0 \text {. }
$$

But the last equation is the same as $\alpha_{12} \bar{\alpha}_{32} \alpha_{34} \bar{\alpha}_{54} \cdots \alpha_{2 n-1,2 n} \bar{\alpha}_{1,2 n}=1$, which we know to be true by Lemma 33 . So $(*)$ has solutions. Hence $\varphi(A)=V A V^{*}$ for every $A$ in $\operatorname{Alg} \mathscr{L}_{2 n}$.

THEOREM 35. If $\varphi: \operatorname{Alg} \mathscr{L}_{2 n} \rightarrow \operatorname{Alg} \mathscr{L}_{2 n}$ is a surjective isometry such that $\varphi(I)=I, \varphi\left(E_{11}\right)=E_{2 i+1,2 i+1}, \varphi\left(E_{22}\right)=E_{2 i, 2 i}, \varphi\left(E_{33}\right)=$ $E_{2 i-1,2 i-1}, \ldots, \varphi\left(E_{2 i-1,2 i-1}\right)=E_{22}, \varphi\left(E_{2 i, 2 i}\right)=E_{11}, \varphi\left(E_{2 i+1,2 i+1}\right)=$ $E_{2 n, 2 n}, \ldots, \varphi\left(E_{2 n, 2 n}\right)=E_{2 i+2,2 i+2}$. Then there exists a unitary operator $W$ such that $\varphi(A)=W A W^{*}$ for all $A$ in $\mathrm{Alg} \mathscr{L}_{2 n}$.

\section{Proof. Let $U_{2 i+1}=D_{2 i+1} \oplus D_{2 n-2 i-1}$.}

Define $\varphi_{1}: \operatorname{Alg} \mathscr{L}_{2 n} \rightarrow \operatorname{Alg} \mathscr{L}_{2 n}$ by $\varphi_{1}(A)=U_{2 i+1} A U_{2 i+1}^{*}$ for every $A$ in $\operatorname{Alg} \mathscr{L}_{2 n}$. where $U_{2 i+1}=U_{2 i+1}^{*}$. Then $\varphi_{1}$ is a surjective isometry because $U_{2 i+1} A U_{2 i+1}$ is in $\operatorname{Alg} \mathscr{L}_{2 n}$ for every $A$ in Alg $\mathscr{L}_{2 n}$. See Example 2. Define $\tilde{\varphi}=\varphi_{1} \circ \varphi$. Then $\tilde{\varphi}\left(E_{i i}\right)=\varphi_{1} \circ \varphi\left(E_{i i}\right)=E_{i i}$ for each $i, i=1,2,3, \ldots, 2 n$. So there exists a unitary operator $V$ such that $\tilde{\varphi}(A)=V A V^{*}$ for every $A$ in $\operatorname{Alg} \mathscr{L}_{2 n}$ by Theorem 34. Since $\tilde{\varphi}(A)=$ $\varphi_{1} \circ \varphi(A)=U_{2 i+1} \varphi(A) U_{2 i+1}^{*}=V A V^{*}$ for every $A$ in $\operatorname{Alg} \mathscr{L}_{2 n}, \varphi(A)=$ $U_{2 i+1}^{*} V A V^{*} U_{2 i+1}$. Set $U_{2 i+1}^{*} V=W$. Then $\varphi(A)=W A W^{*}$ for every $A$ in $\mathrm{Alg} \mathscr{L}_{2 n}$.

THEOREM 36. If $\varphi: \operatorname{Alg} \mathscr{L}_{2 n} \rightarrow \operatorname{Alg} \mathscr{L}_{2 n}$ is a surjective isometry such that $\varphi(I)=I, \varphi\left(E_{11}\right)=E_{2 i+1,2 i+1}$,

$$
\begin{aligned}
\varphi\left(E_{22}\right) & =E_{2 i+2,2 i+2}, \ldots, \varphi\left(E_{2 n-2 i, 2 n-2 i}\right) \\
& =E_{2 n, 2 n}, \varphi\left(E_{2 n-2 i+1,2 n-2 i+1}\right) \\
& =E_{11}, \ldots, \varphi\left(E_{2 n, 2 n}\right)=E_{2 i, 2 i},
\end{aligned}
$$


then there exists a unitary operator $W$ such that $\varphi(A)=W A W^{*}$ for every $A$ in $\operatorname{Alg} \mathscr{L}_{2 n}$.

Proof. Let

$$
V_{2 n-2 i+1}=\left[\begin{array}{cc}
0 & I_{2 n-2 i} \\
I_{2 i} & 0
\end{array}\right]
$$

Define $\varphi_{1}: \operatorname{Alg} \mathscr{L}_{2 n} \rightarrow \operatorname{Alg} \mathscr{L}_{2 n}$ by $\varphi_{1}(A)=V_{2 n-2 i+1} A V_{2 n-2 i+1}^{*}$ for every $A$ in $\operatorname{Alg} \mathscr{L}_{2 n}$. Then since $V_{2 n-2 i+1} A V_{2 n-2 i+1}^{*}$ and $V_{2 n-2 i+1}^{*} A V_{2 n-2 i+1}$ are in $\operatorname{Alg} \mathscr{L}_{2 n}$ for every $A$ in $\operatorname{Alg} \mathscr{L}_{2 n}, \varphi_{1}$ is a surjective isometry. See Example 3. Define $\tilde{\varphi}=\varphi_{1} \circ \varphi$. Then $\tilde{\varphi}\left(E_{i i}\right)=E_{i i}^{*}$ for each $i$, $i=1,2, \ldots, 2 n$. So there exists a unitary operator $U$ such that $\tilde{\varphi}(A)=$ $U A U^{*}$ for every $A$ in $\operatorname{Alg} \mathscr{L}_{2 n}$ by Theorem 34. Since $\tilde{\varphi}(A)=\varphi_{1} \circ$ $\varphi(A)=V_{2 n-2 i+1} \varphi(A) V_{2 n-2 i+1}^{*}=U A U^{*}$ for every $A$ in $\operatorname{Alg} \mathscr{L}_{2 n}, \varphi(A)=$ $V_{2 n-2 i+1}^{*} U A U^{*} V_{2 n-2 i+1}$ for every $A$ in $\operatorname{Alg} \mathscr{L}_{2 n}$. Set $V_{2 n-2 i+1}^{*} U=W$. Then $\varphi(A)=W A W^{*}$ for every $A$ in $\operatorname{Alg} \mathscr{L}_{2 n}$.

THEOREM 37. If $\varphi: \operatorname{Alg} \mathscr{L}_{2 n} \rightarrow \operatorname{Alg} \mathscr{L}_{2 n}$ is a surjective isometry such that $\varphi(I)=I, \varphi\left(E_{11}\right)=E_{11}, \varphi\left(E_{22}\right)=E_{2 n, 2 n}, \varphi\left(E_{33}\right)=E_{2 n-1,2 n-1}, \ldots$, $\varphi\left(E_{2 i-1,2 i-1}\right)=E_{2 n-(2 i-1-2), 2 n-(2 i-1-2)}, \ldots, \varphi\left(E_{2 n, 2 n}\right)=E_{22}$, then there exists a unitary operator $W$ such that $\varphi(A)=W A W^{*}$ for every $A$ in $\operatorname{Alg} \mathscr{L}_{2 n}$.

Proof. Let $U=D_{1} \oplus D_{2 n-1}$. Define $\varphi_{1}: \operatorname{Alg} \mathscr{L}_{2 n} \rightarrow \operatorname{Alg} \mathscr{L}_{2 n}$ by $\varphi_{1}(A)=U A U^{*}$ for every $A$ in $\operatorname{Alg} \mathscr{L}_{2 n}$, where $U=U^{*}$. Then $\varphi_{1}$ is a surjective isometry because $U A U$ is in $\operatorname{Alg} \mathscr{L}_{2 n}$ for every $A$ in $\operatorname{Alg} \mathscr{L}_{2 n}$. Define $\tilde{\varphi}=\varphi_{1} \circ \varphi$. Then $\tilde{\varphi}\left(E_{i i}\right)=\varphi_{1} \circ \varphi\left(E_{i i}\right)=E_{i i}$ for each $i, i=1,2, \ldots, 2 n$. So there exists a unitary operator $V$ such that $\tilde{\varphi}(A)=V A V^{*}$ for every $A$ in $\operatorname{Alg} \mathscr{L}_{2 n}$ by Theorem 34. Since $\tilde{\varphi}(A)=\varphi_{1} \circ \varphi(A)=U \varphi(A) U^{*}=V A V^{*}$ for every $A$ in $\operatorname{Alg} \mathscr{L}_{2 n}, \varphi(A)=$ $U^{*} V A V^{*} U$. Set $U^{*} V=W$. Then $\varphi(A)=W A W^{*}$ for every $A$ in $\operatorname{Alg} \mathscr{L}_{2 n}$.

The last three theorems exhaust all possible cases where $\varphi\left(E_{11}\right)=$ $E_{k k}$ and $k$ is an odd number. Then the last three theorems show that there exists a diagonal unitary operator $U$ such that $\varphi(A)=U A U^{*}$ for every $A$ in $\operatorname{Alg} \mathscr{L}_{2 n}$. If $k$ is an even number, then Theorem 26 and the last three theorems show that there exists a unitary operator $W$ and a conjugation $J$ such that $\varphi(A)=J W A^{*} W^{*} J$ for each $A$ in $\operatorname{Alg} \mathscr{L}_{2 n}$. If $\varphi(I)=U \neq I$, then the reduction following Lemma 8 shows that there exists a unitary $U$ so that the isometry $\hat{\varphi}(A)=U^{*} \varphi(A)$ has one of the above two forms. Thus the main theorem has been proved. 


\section{REFERENCES}

[1] W. Arveson, Operator algebras and invariant subspaces, Ann. of Math., 100 (1974), 443-532.

[2] F. Gilfeather and R. L. Moore, Isomorphisms of certain CSL algebras, J. Funct. Anal., 67 (1986), 264-291.

[3] F. Gilfeather and D. Larson, Commutants modulo the compact operators of certain CSL algebras, Topics in Modern Operator Theory, Advances and Applications, 2, Birkhauser (1982).

[4] F. Gilfeather, Derivations on certain CSL algebras, J. Operator Theory, 11(1) (1984), 91-108.

[5] F. Gilfeather, A. Hopenwasser and D. Larson, Reflexive algebras with finite width lattices; tensor products, cohomology, compact perturbations:, J. Funct. Anal., 55 (1984), 176-199.

[6] P. R. Halmos, A Hilbert Space Problem Book, Second Edition, Springer-Verlag, New York (1982).

[7] A. Hopenwasser, C. Laurie and R. L. Moore, Reflexive algebras with completely distributive subspace lattices, J. Operator Theory, 11 (1984), 91-108.

[8] R. Kadison, Isometries of operator algebras, Ann. of Math., 54(2) (1951), 325338.

[9] W. Longstaff, Strongly reflexive lattices, J. London Math. Soc., 2(11) (1975), 491-498.

[10] R. L. Moore and T. T. Trent, Isometries of nest algebra, preprint, (1987).

[11] H. L. Royden, Real Analysis, The Macmillan Company, New York, (1968).

Received November 5, 1987 and in revised form June 20, 1988. This paper is the author's Ph.D. thesis, written at the University of Alabama, under the direction of R. L. Moore.

KEIMYUNG UNIVERSITY

DAEGU, 704-200, Korea 



\section{PACIFIC JOURNAL OF MATHEMATICS EDITORS}

\author{
V. S. VARADARAJAN \\ (Managing Editor) \\ University of California \\ Los Angeles, CA 90024-1555-05 \\ Herbert Clemens \\ University of Utah \\ Salt Lake City, UT 84112 \\ THOMAS ENRIGHT \\ University of California, San Diego \\ La Jolla, CA 92093
}

R. FINN

Stanford University

Stanford, CA 94305

HeRMANN FlaschKa

University of Arizona

Tucson, AZ 85721

VAUGHaN F. R. Jones

University of California

Berkeley, CA 94720

STEVEN KeRCKHOFF

Stanford University

Stanford, CA 94305

\author{
ROBION KIRBY \\ University of California \\ Berkeley, CA 94720 \\ C. C. MOORE \\ University of California \\ Berkeley, CA 94720 \\ HAROLD STARK \\ University of California, San Diego \\ La Jolla, CA 92093
}

\section{ASSOCIATE EDITORS}
R. ARENS
E. F. BECKENBACH
B. H. NEUMANN
F. WOLF
K. YoshidA (1906-1982)

\section{SUPPORTING INSTITUTIONS}

UNIVERSITY OF ARIZONA

UNIVERSITY OF BRITISH COLUMBIA

CALIFORNIA INSTITUTE OF TECHNOLOGY

UNIVERSITY OF CALIFORNIA

MONTANA STATE UNIVERSITY

UNIVERSITY OF NEVADA, RENO

NEW MEXICO STATE UNIVERSITY

OREGON STATE UNIVERSITY
UNIVERSITY OF OREGON

UNIVERSITY OF SOUTHERN CALIFORNIA

STANFORD UNIVERSITY

UNIVERSITY OF HAWAII

UNIVERSITY OF TOKYO

UNIVERSITY OF UTAH

WASHINGTON STATE UNIVERSITY

UNIVERSITY OF WASHINGTON 


\section{Pacific Journal of Mathematics}

Vol. 140, No. $1 \quad$ September, 1989

Michel Brestovski, Algebraic independence of solutions of differential

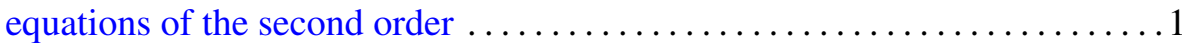

Bohumil Cenkl, Cohomology operations from higher products in the de

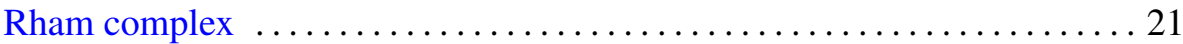

Gustavo Corach and Daniel Suarez, Generalized rational convexity in

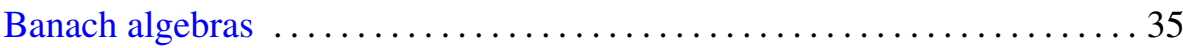

Keresztély Corrádi and Sándor Szabó, A new proof of Rédei’s theorem . . . 53

Steven R. Costenoble and Stefan Waner, Equivariant orientations and

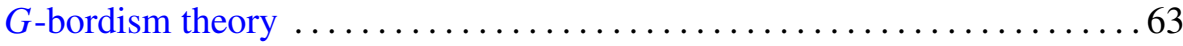

Angel Granja, Apéry basis and polar invariants of plane curve

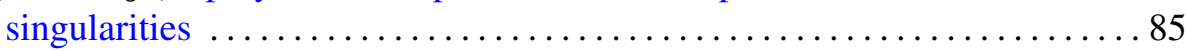

Young Soo Jo, Isometries of tridiagonal algebras .................. 97

Ronald Leslie Lipsman, Harmonic analysis on exponential solvable homogeneous spaces: the algebraic or symmetric cases $\ldots \ldots \ldots \ldots \ldots 117$

Erich Miersemann, On the behaviour of capillaries at a corner 149

Marian Nowak, On the finest Lebesgue topology on the space of essentially

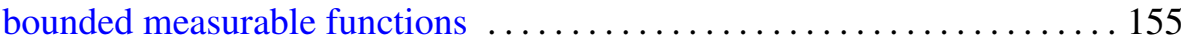

Pascal J. Thomas, Hardy interpolating sequences of hyperplanes ........ 163

H. Bevan Thompson, Differentiability properties of subfunctions for second order ordinary differential equations 